\title{
Perfil dos alunos usuários de lentes de contato do curso de Medicina da Universidade Estadual Paulista - UNESP - Botucatu
}

\author{
Profile of medical students from the Universidade Estadual Paulista - UNESP - \\ Botucatu, who wear contact lenses
}

\author{
Vanessa Goncalves Vidotti \\ Amélia Kamegasawa ${ }^{2}$
}

Trabalho realizado no Departamento de Oftalmologia, Otorrinolaringologia e Cirurgia de Cabeça e Pescoço da Faculdade de Medicina de Botucatu da Universidade Estadual Paulista - UNESP - Botucatu (SP) - Brasil.

${ }^{1}$ Acadêmica do $5^{\circ}$ ano da Faculdade de Medicina da Universidade Estadual Paulista - UNESP - Botucatu (SP) - Brasil.

${ }^{2}$ Professora Assistente Doutora do Departamento de Oftalmologia, Otorrinolaringologia e Cirurgia de Cabeça e Pescoço da Faculdade de Medicina da UNESP Botucatu (SP) - Brasil.

Endereço para correspondência: Amélia Kamegasawa. Departamento de OFT/ORL/CCP da Faculdade de Medicina de Botucatu da Universidade Estadual Paulista - UNESP. Rubião Júnior, s/n - Botucatu (SP)

CEP 18618-970

E-mail: kameg@fmb.unesp.br

Recebido para publicação em 30.03.2004

Versão revisada recebida em 23.09.2005

Aprovação em 02.12.2005

\begin{tabular}{|c|}
\hline RESUMO \\
\hline $\begin{array}{l}\text { Objetivo: Analisar o perfil dos usuários de lentes de contato entre os } \\
\text { estudantes de medicina da Faculdade de Medicina de Botucatu da Univer- } \\
\text { sidade Estadual Paulista - UNESP - Botucatu (SP), quanto à ametropia, à } \\
\text { aquisição, aos cuidados e às complicações. Métodos: Foi aplicado um } \\
\text { questionário aos alunos do } 1^{\circ} \text { ao } 6^{\circ} \text { ano de Medicina da Faculdade de } \\
\text { Medicina de Botucatu da Universidade Estadual Paulista - UNESP - } \\
\text { Botucatu (SP), perfazendo um estudo analítico transversal. Resultados: } \\
\text { Foram } 566 \text { entrevistados. Destes, } 155 \text { ( } 27,4 \%) \text { usavam lente de contato. } \\
\text { Das lentes de contato usadas, } 94,8 \% \text { eram gelatinosas, e em } 98,7 \% \text { o grau } \\
\text { era negativo. O descarte dessas lentes era mensal em } 46,9 \% \text { dos entrevis- } \\
\text { tados e } 63,9 \% \text { a utilizavam, em média, } 14 \text { horas/dia. Faziam aquisição da } \\
\text { lente de contato em óptica ou farmácia } 14,1 \% \text { dos estudantes. Faziam a } \\
\text { higiene ao colocá-las e ao retirá-las dos olhos, } 46,5 \% \text {. As complicações } \\
\text { referentes aos olhos vermelhos eram } 51,0 \% \text {; } 44,5 \% \text {, embaçamento; e } \\
42,6 \% \text {, sensação de corpo estranho, além de } 4 \text { estudantes relatarem } \\
\text { "ceratite". Conclusões: A maioria dos alunos utilizava lentes hidrofílicas, } \\
\text { descarte mensal e grau negativo. A adaptação inadequada, aquisição de } \\
\text { lentes de contato em ópticas ou farmácias e complicações graves ocorre- } \\
\text { ram, mesmo nos usuários socioculturalmente considerados diferenciados. } \\
\text { Em vista disso, demanda a extrema necessidade de maior atenção na } \\
\text { abordagem do tema em questão, a fím de prevenir o comprometimento } \\
\text { visual permanente. }\end{array}$ \\
\hline
\end{tabular}

Descritores: Lentes de contato/efeitos adversos; Acomodação ocular; Infecções oculares bacterianas; Ceratite; Estudantes de medicina

\section{INTRODUÇÃO}

Desde o advento da lente de contato (LC), a melhora de sua qualidade, o desenvolvimento de produtos para limpeza e conservação e a facilidade de aquisição pela população o número de usuários cresce diariamente ${ }^{(1)}$.

Segundo o relato anual de 1998 sobre LC, estima-se que existam 33 milhões de usuários nos Estados Unidos ${ }^{(2)}$. No entanto, o grande número de usuários faz com que cresça também o número de indivíduos com complicações oculares devido ao uso inadequado. Dentre estas complicações, a ceratite microbiana tem sido relatada ${ }^{(3-6)}$.

Mas não somente o uso inadequado predispõe a complicações, já que descrições apontam LC de uso contínuo como fator de risco significativo para 
o desenvolvimento de ceratite microbiana ${ }^{(7-9)}$. Apesar disto, Nilsson et al., estabeleceram que existe tendência à redução em úlcera de córnea associada ao uso de LC na sua instituição ${ }^{(10)}$.

O sucesso do uso de LC requer a escolha de uma lente adequada ao olho e demanda que o paciente tenha condições de compreender e se adaptar ao uso e limitações das lentes, bem como à aderência ao seu manuseio. O paciente deve ainda estar informado em relação à conservação, ao esquema de uso e à identificação da sintomatologia de perigo, ciente do pronto acesso a cuidados especializados ${ }^{(11)}$.

Este estudo teve como objetivo avaliar o perfil de usuários de lentes de contato entre os estudantes da Faculdade de Medicina da UNESP - Botucatu, analisando os seguintes fatores através de um estudo analítico transversal: a) ametropia; b) aquisição da LC; c) cuidados com a LC; d) complicações apresentadas com o uso da LC.

\section{MÉTODOS}

Formulou-se um questionário, com questões de múltipla escolha e questões dissertativas curtas e diretas. Este foi distri- buído aos estudantes de medicina do primeiro ao sexto ano em 2003, os quais preencheram a avaliação, preservando-se o anonimato. Todos foram informados do caráter do trabalho e assinaram o termo de consentimento aprovado pela comissão de ética. $\mathrm{O}$ questionário e o termo de consentimento seguem abaixo.

\section{TERMO DE CONSENTIMENTO LIVRE E ESCLARECIDO}

$\mathrm{Eu}$, $R G$

concordo em participar da pesquisa "Perfil dos alunos de curso de medicina da UNESP-BOTUCATU, usuários de lentes de contato", aos cuidados da Dra. Amélia Kamegasawa. Informo que estou consciente e perfeitamente esclarecido quanto ao questionário que responderei e que estes dados serão sigilosos. Eu entendo que minha participação é voluntária e que tenho direito de retirar minha autorização a qualquer momento sem nenhum prejuízo. Concordo com a publicação dos resultados em reuniões ou revistas científicas. Estou também ciente que se não me sentir atendido poderei procurar a pesquisadora (Dra. Amélia Kamegasawa), conforme identificação e endereço contidos neste termo.

Nome: RG:

Assinatura: Data: $23 / 06 / 2003$

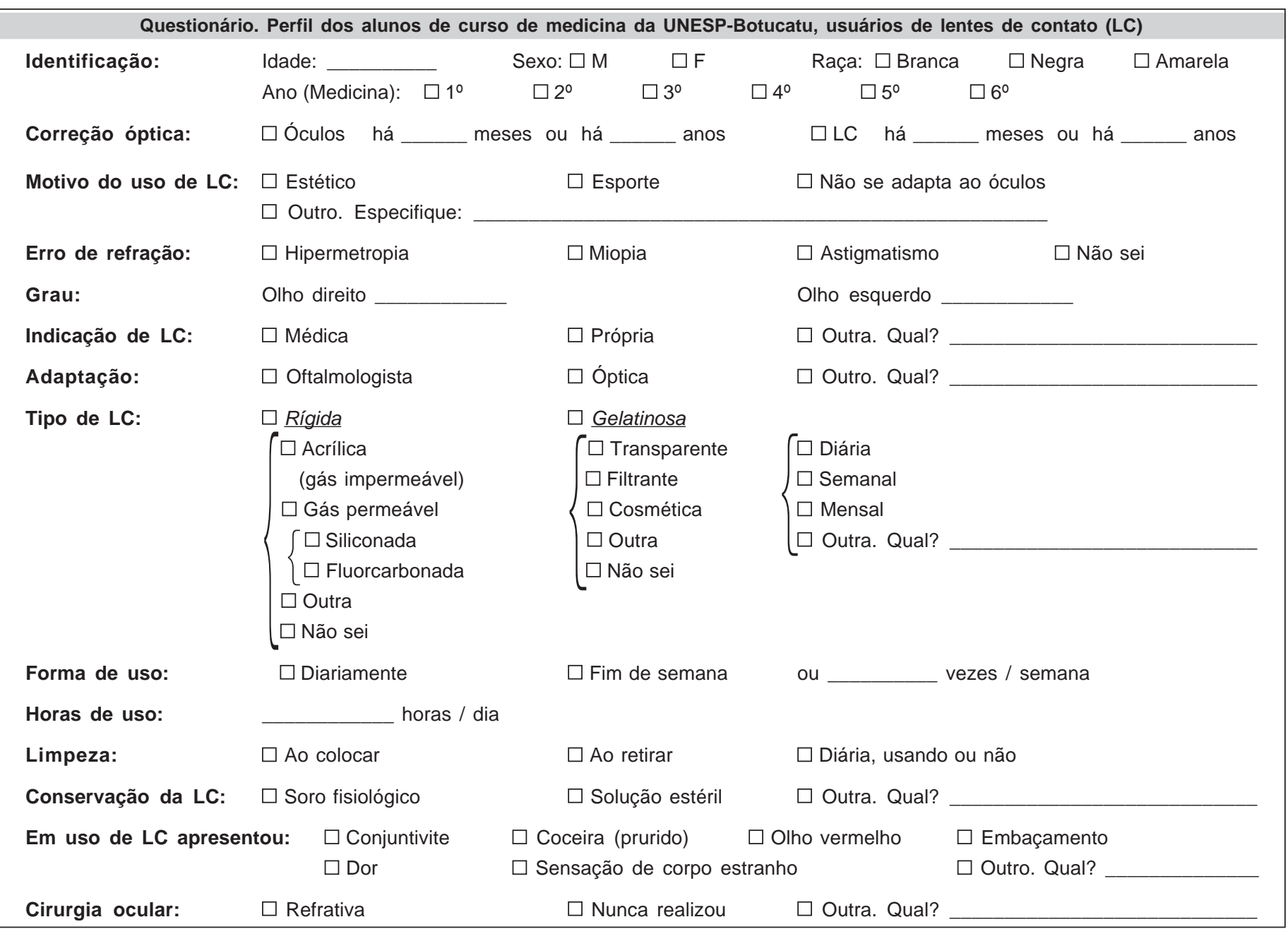




\section{RESULTADOS}

De 566 estudantes do curso de Medicina, 155 (27,4\%) eram usuários de lentes de contato e responderam ao questionário aplicado.

A faixa etária mais prevalente situou-se entre 21 e 24 anos $(67,7 \%)$, sendo a maioria brancos $(75,5 \%)$ e do sexo feminino $(62,6 \%)$ (Gráfico 1).

Os usuários de LC também faziam uso de óculos, sendo este mais precoce. A lente mais utilizada foi a gelatinosa $(94,8 \%)$ com descarte mensal $(46,9 \%)$ e $63,9 \%$ utilizavam a LC 12 a 16 horas por dia.

Os motivos de uso da LC mais citados foram o estético $(58,3 \%)$ e para a prática de esportes $(28,2 \%)$. Os erros de refração prevalentes foram miopia (49\%) e astigmatismo miópico composto $(49,7 \%$ ) (Gráfico 2 ). A ametropia variou de -1 a $-4 \mathrm{D}(66,2 \%)$.

A indicação e adaptação da LC foram realizadas, principalmente por oftalmologista, embora $14,1 \%$ adaptassem em óptica ou farmácia (Gráficos 3 e 4).

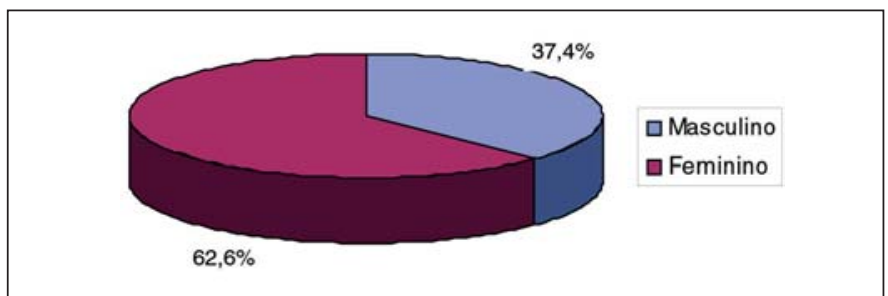

Gráfico 1 - Usuários de lente de contato segundo o sexo

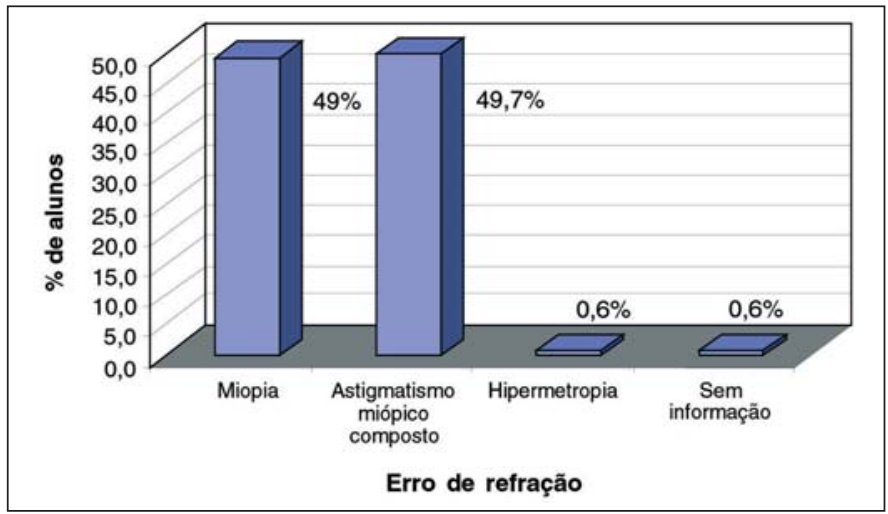

Gráfico 2 - Erro de refração apresentado pelos usuários de lente de contato

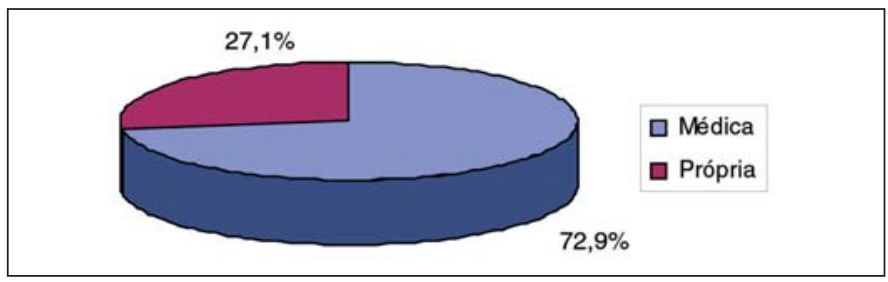

Gráfico 3 - Indicação da lente de contato
A higienização da LC, em 27,7\% dos entrevistados, era feita ao colocá-la; $20,6 \%$, ao retirá-la; 46,5\%, ao colocá-la e retirá-la; e somente $3,9 \%$ faziam a limpeza diária mesmo quando não a utilizavam (Gráfico 5). A grande maioria (91\%) as conservava em soluções estéreis.

Questionados quanto às complicações, apenas 16,8\% responderam que nunca as havia apresentado, sendo que 92,6\% destes adaptaram a LC no oftalmologista. Dentre as complicações mais citadas encontrou-se: olho vermelho (51\%), embaçamento $(44,5 \%)$ e sensação de corpo estranho (42,6\%), (Tabela 1), além do relato de "ceratite" por 4 entrevistados $(2,6 \%)$. Nenhum dos entrevistados havia realizado cirurgia ocular prévia.

\section{DISCUSS ÃO}

A faixa etária mais prevalente estudada situou-se entre 21 e 24 anos, com predomínio de usuários do sexo feminino $(62,6 \%)$, comparável com outro estudo baseado em entrevista com estudantes e trabalhadores da saúde, sobre auto-avaliação quanto ao uso e cuidado com a LC, o qual encontrou predomínio de mulheres $(69,2 \%)$ usuárias de LC, com uma média de idade de

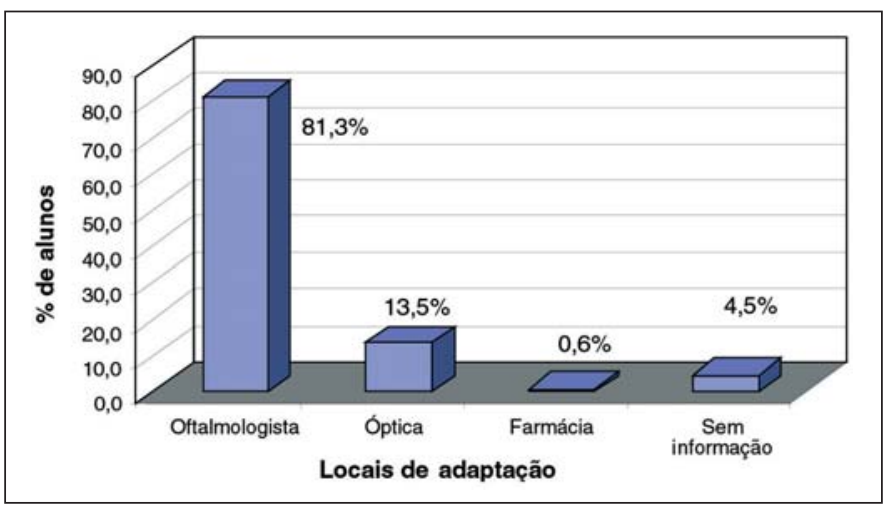

Gráfico 4 - Local de adaptação da lente de contato

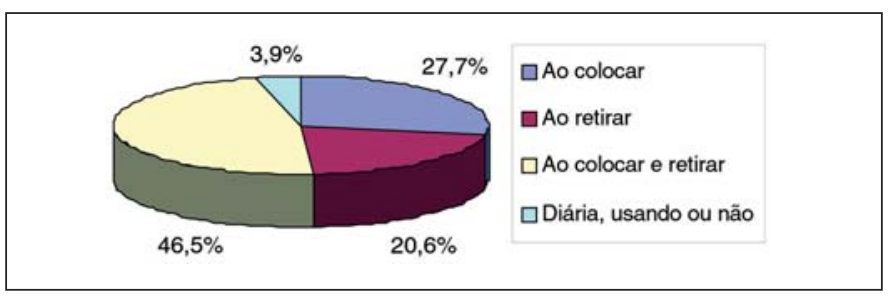

Gráfico 5 - Forma de limpeza da lente de contato

\begin{tabular}{|lc|}
\hline Tabela 1. Complicações apresentadas com o uso de lentes de contato \\
Complicações & $\%$ de alunos \\
Olho vermelho & 51,0 \\
Embaçamento & 44,5 \\
Sensação de corpo estranho & 42,6 \\
Prurido & 29,7 \\
Dor & 17,4 \\
\hline
\end{tabular}


23,5 $\operatorname{anos}^{(12)}$. Acompanhando a tendência da literatura, a lente hidrofílica foi a preferida pelos entrevistados ${ }^{(13)}$.

Em estudo realizado no Estado de São Paulo, que objetivou a avaliação das condições de adaptação e venda de LC em ópticas, observou-se a inadequação na qual é realizada a adaptação do produto, sem seleção, controle ou orientação aos usuários ${ }^{(14)}$. Isso demonstra o grande risco ao qual $14,1 \%$ dos estudantes entrevistados neste trabalho estavam sendo submetidos. Esses dados corroboram com o fato de que a grande maioria dos entrevistados que relatou nunca ter apresentado complicações com o uso da LC, realizou adaptação através de um oftalmologista $(92,6 \%)$. Além disso, alguns autores, declararam que $80 \%$ das complicações apresentadas com o uso de LC podem ser relacionadas com a compreensão deficiente das recomendações quanto ao uso e à manutenção do produto $^{(15)}$.

Em relação aos cuidados com as LC, observou-se que apenas $46,5 \%$ dos entrevistados faziam a limpeza da LC ao colocá-la e retirá-la do olho e $91,0 \%$ a conservava em solução estéril. Levando em consideração o processo de manutenção da LC recomendado como profilaxia para infecções ${ }^{(16)}$, percebe-se que os cuidados realizados por entrevistados neste trabalho estão aquém dos esperados, o que se configura como mais um fator de risco para complicações.

Por fim, quanto às sintomatologias referidas como complicações, embora a grande maioria não apresente dano imediato para a visão, devem ser avaliadas prontamente por um oftalmologista, pois podem ser sintomas precoces de infecções ou de alterações da superfície ocular, predispondo a complicações mais graves ${ }^{(16)}$. Além disso, não podemos deixar de citar os quatro casos de ceratites apresentadas na amostra estudada. Apesar de serem poucos casos e não terem sido especificados se infecciosos ou não, foram abordados nesta discussão já que as ceratites representam a mais séria complicação do uso de LC com risco de cegueira ${ }^{(17-18)}$. Estudos demonstram que aproximadamente $21 \%$ dos casos de ceratite ulcerativa são atribuídos ao uso de $\mathrm{LC}^{(14-15)}$. O observado neste trabalho de que mesmo em usuários diferenciados, como estudantes de medicina, ocorreu adaptação inadequada da LC, ratifica a necessidade de maior atenção na abordagem do tema pelos especialistas e órgãos de saúde pública a fim de prevenir cegueira, considerando o aumento de usuários de LC.

\section{CONCLUSÕES}

A correção óptica utilizada foi preferencialmente a LC do tipo gelatinosa de descarte mensal para correção de graus negativos. Muitos dos entrevistados, apesar de pertencerem a um nível socioeconômico e sociocultural considerado diferenciado, adquiriam as $\mathrm{LC}$ em ópticas ou farmácias. O cuidado insuficiente com a LC como profilaxia para infecções, bem como adaptação inadequada foram observadas, o que submetiam os entrevistados ao risco de complicações.

\section{AGRADECIMENTOS}

Dra. Maria Rosa Bet de Moraes Silva e Dra. Silvana Artioli Schellini.

\section{ABSTRACT}

Purpose: To analyze the ametropy, way of acquisition, the care and complications related to contact lens wear among medical students from Faculdade de Medicina de Botucatu da Universidade Estadual Paulista - UNESP - Botucatu (SP). Methods: Questionnaire applied to students from the $1^{\text {st }}$ to the $6^{\text {th }}$ year of the Medical school of the Faculdade de Medicina de Botucatu da Universidade Estadual Paulista - UNESP - Botucatu (SP), forming an analytical cross-sectional survey. Results: $27.4 \%$ of the students use contact lenses, and among them $94.8 \%$ use soft contact lenses and $98 \%$ have negative degree. $46.9 \%$ discard their contact lens monthly. $63.9 \%$ have an average daily use of 14 hours, $14.1 \%$ acquire the contact lenses from optician stores or at pharmacies and $46.5 \%$ of the students clean their contact lenses when they put them on and take them off. Regarding complications, $51.0 \%$ reported ocular redness; $44.5 \%$, blurred vision; and $42.6 \%$, foreign body sensation, whereas only 4 students reported "keratitis". Conclusion: We concluded that the inadequate adaptation that occurs even in users knowledgeable about this issue such as medical students, has increased the risk of complications. This fact indicates that more attention must be paid to contact lens care in order to prevent permanent visual deficit.

Keywords: Contact lenses/adverse effects; Accommodation, ocular; Eye infections, bacterial; Keratitis/etiology; Students, medical

\section{REFERÊNCIAS}

1. Coral-Ghanem C, Kara-José N. Lente de Contato na Clinica Oftalmológica. Joinville, Santa Catarina: Sociedade Brasileira de Lente de Contato e Córnea, 1985 .

2. Barr JT. The 1998 annual report on contact lenses. Contact Lens Spectr. 1999; $15: 25-8$.

3. Stern GA. Contact lens associated bacterial keratitis: past, present and future. CLAO J. 1998;24(1):52-6.

4. Poggio EC, Glynn RJ, Schein OD, Seddon JM, Shannon MJ, Scardino VA, et al. The incidence of ulcerative keratitis among users of daily-wear and extended -wear soft contact lenses. N Engl J Med. 1989;321(12):779-83. Comment in: N Engl J Med. 1989;321(12):824-6; N Engl J Med. 1990;322(10):700-1.

5. Laibson PR, Cohen EJ, Rajpal RK. Conrad Berens Lecture. Corneal ulcers related to contact lenses. CLAO J. 1993;19(1):73-8.

6. Grant T, Chong MS, Vajdic C, Swarbrick HA, Gauthier C, Sweeney DF, et al. Contact lens induced peripheral ulcers during hydrogel contact lens wear. CLAO J. 1998;24(3):145-51. Comment in: CLAO J. 1998;24(3):134-136.

7. Cohen EJ, Fulton JC, Hoffman CJ, Rapuano CJ, Laibson PR. Trends in contact lens-associated corneal ulcers. Cornea. 1996;15(6):566-70.

8. Schein OD, Buehler PO, Stamler JF, Verdier DD, Katz J. The impact of overnight wear on the risk of contact lens-associated ulcerative keratitis. Arch Ophthalmol. 1994;112(2):186-90. Comment in: Arch Ophthalmol. 1994; 112(2):173-4; Arch Ophthalmol. 1994;112(11):1404-6. 
9. Schein OD, Glynn RJ, Poggio EC, Seddon JM, Kenyon KR. The relative risk of ulcerative keratitis among users of daily-wear and extended-wear soft contact lenses. A case-control study. Microbial Keratitis Study Group. N Engl J Méd. 1989;321(12):773-8. Comment in: N Engl J Méd. 1989;321(12):824-6; N Engl J Méd. 1990;322(10):700-1.

10. Nilsson SE, Montan PG. The hospitalized cases of contact lens induced keratitis in Sweden and their relationship to lens type and wearing schedule: result of a three year retrospective study. CLAO J. 1994;20(2):97-101. Comment in: CLAO J. 1994;20(2):86-7.

11. Coral-Ghanem C, Kara-José N. Complicações associados ao uso de lente de contato in: Coral-Ghanem C, Kara-José N. Lente de Contato na Clinica Oftalmológica. $2^{\underline{a}}$ ed. Rio de Janeiro: Cultura Médica: 1998, 133-6.

12. Oliveira PR, Temporini-Nastari ER, Ruiz Alves M, Kara-José N. Self-evaluation of contact lens wearing and care by college students and health care workers. Eye Contact Lens. 2003;29(3):164-7.
13. Minguini N, Coelho RP, Serpa JF, Kara-José N, Holzchuh N. Características do uso de óculos e de lente de contato em uma comunidade universitária. Arq Bras Oftalmol. 1994;57:126-8.

14. Kara-José AC, Cunha KG, Malta JBNS, Gomes ACM, Novelli FJD. Condições de adaptação e venda de lentes de contato em óticas do estado de São Paulo. Arq Bras Oftalmol. 2001;64(5):429-35.

15. Ky W, Scherick K, Stenson S. Clinical survey of lens care in contact lens patients. CLAO J. 1998;24(4):216-9. Comment in: CLAO J. 1998;24(4):194.

16. Freitas D. Profilaxia da infecção durante o uso de lentes de contato. Universo Visual. 2003;11:16-8.

17. Mela EK, Giannelou IP, John KX, Sotirios GP. Ulcerative keratitis in contact lens wearers. CLAO. 2003;29(4):207-9.

18. Galentine PG, Cohen EJ, Laibson PR, Adams CP, Michaud R, Arentsen JJ. Corneal ulcers associated with contact lens wear. Arch Ophthalmol. 1984; 102(6):891-4.

\title{
XIII Simpósio Internacional da Santa Casa de Sãa Paula
}

\section{8 a 10 de Junho de 2006}

\section{Frel Caneca Shopping \& Convention Center}

\author{
5ão Paula - 5P
}

INFORMRÇÕES: JUE Camunicaçãa e Eventos

Tels.: (11) 5084-5284/5084-9174

Fax: (11) 5574-8261

E-mail: jdecomev@uol.com.br 